\title{
Can aqueous two-phase micellar systems (ATPMS) be effective platforms for the encapsulation of hydrophobic drugs like curcumin as a model?
}

\author{
Mayra S. H. Santos*, Mayra C. C. Câmara, Isabelle S. Kurnik, Jorge F. B. Pereira, Priscila G. Mazzola, and André \\ M. Lopes.
}

\begin{abstract}
Polymeric micelles (PMs) obtained by aqueous two-phase micellar systems (ATPMS) hold a large potential for cloud point (CP) extraction of biopharmaceuticals. However, the number of studies evaluating their capability to encapsulate drugs are still scarce, hence limiting their employment in formulations/systems. In this work, the effect of different choliniumbased ionic liquids (ILs) upon the clouding behavior of PEG-PPG-PEG amphiphilic triblock copolymer (Pluronic F68) was evaluated. The influence of the ILs on the binodal curves was dependent on the concentration and type of ILs added to the ATPMS. In summary, it was observed an increase of the biphasic region and the decrease of CP values (i.e. close to room temperature $\sim 25$ to $30^{\circ} \mathrm{C}$ ) by adding ILs to the copolymer aqueous solution.
\end{abstract}

Key words: Polymeric micelles (PMs), ionic liquids (ILs), cloud point (CP).

\section{Introduction}

Amphiphilic block copolymers (ABCs) can selfassemble into polymeric micelles (PMs) in aqueous media. PMs have been widely studied in the field of nanomedicine, biomedicine, and pharmacy. These systems can also be obtained by the formation of simple aqueous micellar two-phase systems (ATPMS), which have a great potential for the separation and/or encapsulation of drugs or biopharmaceuticals by cloud point (CP) extraction. The ATPMS are thermoreversible systems, which present two macroscopic liquid phases with distinct concentrations of $A B C s$, one rich in $A B C s$ and other colloidal components, known as coacervate (micellerich phase), in equilibrium with another coexisting liquid phase relatively diluted, poor in $\mathrm{ABCs}$ (micelle-poor phase), above a temperature known as the CP. The CPs identify the boundary between the single-phase and biphasic regions, forming a binodal curve. Interestingly, CPs can be properly manipulated by the addition of adjuvants, i.e., ionic liquids (ILs) and/or salts (Vicente et al., 2017; Wang and Wang, 20014). Particularly, the use of ILs as adjuvants allows a proper control of the separation temperature by changing the phase separation behavior, namely, to increase or decrease the biphasic region, as well as to modify inter- and intra-micellar interactions, influencing then the drugs encapsulation ability.

This approach is of upmost importance in the nanotechnological field, hence it allows the development of innovative and alternative formulation for delivering of lipophilic drugs with low solubility in the aqueous environment. For example, curcumin (CCM) is a natural polyphenolic compound with antioxidant, antimicrobial, and antitumor properties, but its hydrophobicity has been limiting its therapeutic application. PMs can thus provide a favorable environment to load the CCM in aqueous environment. Taking this into consideration, herein, the effect of different cholinium-based ionic liquids \{choline chloride $([\mathrm{Ch}] \mathrm{Cl})$, choline acetate $([\mathrm{Ch}][\mathrm{Ac}])$, choline propanoate ([Ch][Prop]), choline butanoate ([Ch][But]), and choline hexanoate $([\mathrm{Ch}][\mathrm{Hex}])\}$ upon the clouding behavior of PEG-PPG-PEG ABCs (Pluronic ${ }^{\circledR}$ F68) was studied.

\section{Results and Discussion}

Firstly, it was determined the binodal curves with and without ILs, for the system composed of the following concentrations of Pluronic ${ }^{\circledR} \mathrm{F} 68$ (wt\%) in PBS buffer (pH 7.4): $0.5,1.5,2.5,5.0,7.5$, and 9.0. The binodal curve of Pluronic ${ }^{\circledR}$ F68 was compared, in Figure 1, with the binodal curves of ATMPS composed of [Ch]Cl $(3.50 \mathrm{M})$, [Ch][Ac] $(3.00 \mathrm{M})$, [Ch][Prop] (2.50 M), [Ch][But] (1.50 M), and [Ch][Hex] (0.50 M). The results from Figure 1 demonstrated that the CP values decreased with the decrease of ILs concentration (M), such as: from 3.5 to $0.50 \mathrm{M}$. Moreover, it was also shown that the reduction of $\mathrm{CP}$ values close to room temperature ( 25 to $30^{\circ} \mathrm{C}$ ), according the IL' hydrophobicity, namely, [Ch] [Hex] > [Ch] [But] $>[\mathrm{Ch}][\mathrm{Prop}]>[\mathrm{Ch}][\mathrm{Ac}]>[\mathrm{Ch}] \mathrm{Cl}$, i.e. the more hydrophobic is the anion of the IL the lower is the $\mathrm{CP}$ and the higher is the biphasic region.

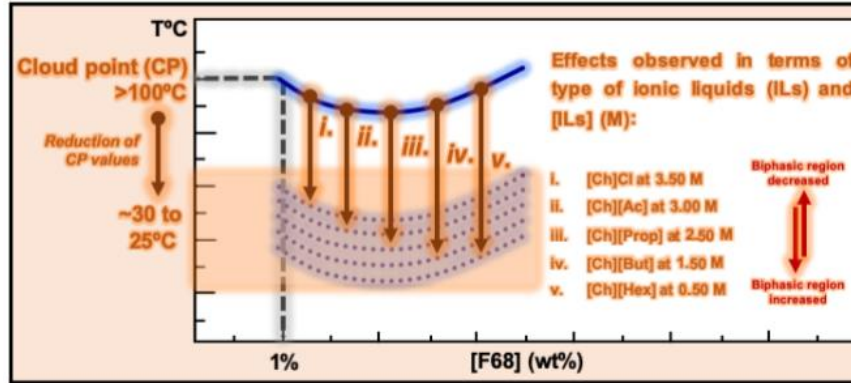

Figure 1. Schematic representation of effect of different cholinium-based ionic liquids [ILs choline chloride ([Ch]Cl), choline acetate ([Ch][Ac]), choline propanoate ([Ch [Prop]), choline butanoate ([Ch[But]), and choline hexanoate ([Ch][Hex]])] on the binodal curves of PEGPPG-PEG amphiphilic triblock copolymers (Pluronic ${ }^{\circledast}$ F68).

\section{Conclusions}

Summing up, the addition of ILs is favorable for the decrease of $\mathrm{CP}$ values and the enlargement of the biphasic region. These results indicate that these systems will facilitate the encapsulation of CCM in PMs at room temperature in the presence of low ILs concentration (i.e., [Ch][Hex] at $0.50 \mathrm{M}$ ). In addition, PMs-based ATPMS approach can avoid the use of very toxic components, such as volatile organic solvents, for the encapsulation of hydrophobic drugs.

\section{Acknowledgement}

The authors are grateful for financial support from FAPESP (São Paulo Research Foundation, Brazil) through the following projects: \#2017/10789-1, \#2018/10799-0, \#2018/05111-9, and \#2019/08549-8.

Vicente, F. A.; Cardoso, I. S.; Sintra, T. E.; Lemus, J.; Marques, E. F.; Ventura S. P.; and Coutinho, J. A. J. Phys. Chem. B. 2017, 121, 8742.

Wang, M.; and Wang, Y. Soft Matter. 2014, 10, 7909. 